\title{
Measurement and analysis of the isomeric cross section ratios for the ${ }^{94} \mathbf{T c}$ nucleus
}

\author{
G. S. Li ${ }^{1,2}$, Y. D. Fang ${ }^{1}{ }^{*}$ X. H. Zhou ${ }^{1}{ }^{\dagger}$ A. Diaz-Torres ${ }^{3}$, A. Rohilla ${ }^{1}$, M. L. Liu ${ }^{1}$, N. T. Zhang ${ }^{1}$, Y. H. Zhang ${ }^{1}$, \\ J. G. Wang ${ }^{1}$, B. S. Gao ${ }^{1}$, Y. H. Qiang ${ }^{1}$, S. Guo ${ }^{1}$, S. C. Wang ${ }^{1}$, Y. Zheng ${ }^{1}$, J. Lubian ${ }^{4}$, and H. O. Soler ${ }^{4}$ \\ 1 Key Laboratory of High Precision Nuclear Spectroscopy and Center for Nuclear Matter Science, \\ Institute of Modern Physics, Chinese Academy of Sciences, \\ Lanzhou, 73000, People's Republic of China \\ 2 School of Nuclear Science and Technology, \\ University of Chinese Academy of Sciences, \\ Beijing 100049, People's Republic of China \\ 3 Department of Physics, University of Surrey, \\ Guildford GU2 7XH, United Kingdom \\ 4 Instituto de Física, Universidade Federal Fluminense, \\ Avenida Litorânea s/n, Gragoatá, Niterói, \\ Rio de Janeiro 24210-340, Brazil
}

(Dated: October 16, 2020)

\begin{abstract}
We report on an isomer yield ratio study of biologically important ${ }^{94} \mathrm{Tc}$ following the fusion of the ${ }^{9} \mathrm{Be}+$ ${ }^{89} \mathrm{Y}$ system, carried out using the offline $\gamma$-ray spectroscopy in continuation to the online activation method. The incident beam energies considered are above the Coulomb barrier for the present study. The PLATYPUS model in conjunction with a potential model calculation was employed to analyze the data. An agreement in the order of magnitude between the experimental data and theoretical predictions has been achieved, by applying a phenomenological approach. The approach was further tested with isomer yield ratios of ${ }^{94} \mathrm{Tc}$ formed through ${ }^{3} \mathrm{He}+{ }^{93} \mathrm{Nb}$ reactions. Possible factors that relate to the isomer yield ratios are also presented.
\end{abstract}

PACS numbers: 25.60.Pj, 25.70.-z

\section{INTRODUCTION}

In recent years, the investigation to Technetium (Tc) isotopes has been a subject of intense experimental and theoretical studies, due to its practical applications, especially in the ${ }^{35}$ 5 nuclear medicine field. For example, the $\gamma$ ray emitted by ${ }^{99} \mathrm{Tc}^{m}$ is widely used for medical diagnostic studies. Different chemical forms have been used for brain, bone, liver, spleen, and kidney imaging as well as blood flow studies [1]. This is benefited from the fact that the ${ }^{99} \mathrm{Tc}^{m}$ has a short half- 40 life $\left(\mathrm{T}_{1 / 2}=6.01 \mathrm{~h}\right)$, and does not remain in the body for a long time. The neutron-deficient Tc isotopes, on the other hand, are also suggested as suitable candidates for medical diagnosis. For instance, the calculated $\gamma$-ray intensities of a ${ }^{95} \mathrm{Tc}^{g}$ and ${ }^{96} \mathrm{Tc}^{g}$ nucleus injected into the human body are $63 \%$ and $70 \%{ }^{45}$ 15 respectively, relative to that of a ${ }^{99} \mathrm{Tc}^{m}$ nucleus, suggesting that the two nuclei can also work as $\gamma$-ray emitters of diagnosis [2]. Other than these nuclei, the ${ }^{94} \mathrm{Tc}^{m}$, with large positron branching ratio (70\%) and medium positron end-point energy (2.4 MeV), is a suitable candidate for Positron Emission To- 50

20 mography, and regarded as an intriguing alternative to many already popular tracers [3-5].

Various nuclear reactions have been employed in the production for Tc isotopes, such as $(p, n),(n, p)$, and deuteriuminduced reactions [6-9]. In addtition, large number of stud- 55 25 ies on the excitation functions related to the isotopes are also available from the literature [10-13]. However, most of these studies are based on the light-ion-induced reactions, and cross section data with heavy-ion-induced reactions are scarce. Maiti et al. [14] have reported on new routs of Tc iso- 60 tions, and the excitation functions were given. Some functions are also available from the ${ }^{12} \mathrm{C}+{ }^{89} \mathrm{Y}$ and ${ }^{9} \mathrm{Be}+{ }^{89} \mathrm{Y}$ reactions $[15,16]$, focusing on the complete fusion $(\mathrm{CF})$ and incomplete fusion (ICF) studies. Still, the data on the heavy-ion-induced reactions and related reaction mechanisms for the production of Tc isotopes are in high demand.

To shed light on the nuclear data of proton-rich Tc isotopes, we have investigated the production of ${ }^{94} \mathrm{Tc}$ formed through the ${ }^{9} \mathrm{Be}+{ }^{89} \mathrm{Y}$ reactions, focusing on the population of different states. Since the ${ }^{9} \mathrm{Be}$ projectile is weakly bound with unusual nucleon distributions, the special (like large probability of breakup before the reaction) can give strong influence to the interaction process. This make the status of residues (e.g., from the CF following breakup) different compared to the fusion reaction cases induced by stable bound projectiles, such as $\alpha,{ }^{12} \mathrm{C}$, etc. In this context, the study will open the opportunity to compare other projectile-target combinations to further explore the underlying reaction dynamics. Specifically, the ${ }^{94} \mathrm{Tc}$ has an isomeric state of $(2)^{+}$and ground state of $7^{+}$, with different characteristic $\gamma$ rays from their decays. This will allow the determination of isomer yield ratios, to get access to the information on the production mechanism, in particular on the angular momentum of excited states of the precursor. We note that the experimental data related to the isomer yield ratio are still scarce in the literature. From the theoretical point of view, different types of models are available to describe the reaction mechanisms, ranging from classical to quantum-mechanical methods [17-21]. Among them, the PLATYPUS model [22] could offer special opportunity to the weakly bound projectile studies, as it uses classical trajectories in conjunction with stochastic breakup. It is meaningful to test the models with available experimental data. 
This paper presents the experimental isomer yield ratios in ${ }^{94} \mathrm{Tc}$, a study of the data by employing the PLATYPUS model combined with potential model calculation. The experimental setup and procedure are described in Sec.II. In Sec.III we present the measured spectra, data analysis, as well as the results. Comparisons of the data with theoretical calculations are shown in Sec.IV. The conclusions drawn from the present study are given in Sec.V.

\section{EXPERIMENTAL DETAILS}

The measurement was performed by the stacked-foil activation technique followed by offline identification of the characteristic $\gamma$ rays of the reaction residues. The detailed descriptions of the setup and the experimental technique can be found in Ref. [23], and only a brief description is given in the present context. The collimated ${ }^{9} \mathrm{Be}$ beam was delivered by the Heavy Ion Research Facility in Lanzhou (HIRFL). Five ${ }^{89} \mathrm{Y}$ targets having thickness $\approx 1.0 \mathrm{mg} / \mathrm{cm}^{2}$ were irradiated

80 for about $12 \mathrm{~h}$ at an average beam current of $13 \mathrm{enA}$. The targets were backed with $\mathrm{Au}$ for reducing the energy of the beam on the subsequent target. The initial energy, delivered by the accelerator, was $50.4 \mathrm{MeV}$. The effective incident energy on each ${ }^{89} \mathrm{Y}$ target was taken as that bombarded at half

85 the target thickness. It was obtained by ATIMA calculation, incorporated within the LISE++ program [24, 25]. Finally, the irradiation of the targets was done in energy range of 45.9 $\sim 50.1 \mathrm{MeV}$, in steps of $1 \sim 1.1 \mathrm{MeV}$. The beam flux was extracted using a precise current-integrator device, from the

90 charge collected at the Faraday cup, which is biased with -400 $\mathrm{V}$ electrode and installed at the back of targets, following the direction of beam flow. The precise current-integrator device can ensure us to extract the beam intensity at regular intervals of $1 \mathrm{~s}$. Additionally, to cross check the beam flux that im-

95 pinged on the targets, two silicon detectors were mounted at $\pm 30^{\circ}$ with respect to the beam line. The elastic scattered ${ }^{9} \mathrm{Be}$ projectiles by a thin Au foil, installed upstream from the targets, were monitored. The two sets of deduced beam currents were found to be in agreement with each other.

After the online beam irradiation, the activated targets were transferred to a separate laboratory for performing offline measurements. A setup formed by five HPGe detector groups was used. Each group comprised of two HPGe detectors placed at an angle of $180^{\circ}$ to each other. This allows the acquisition of data possible both in singles as well as in $\gamma-\gamma$ coincidence mode at the same instance, by placing the activated targets at the optimized distances. Further to reduce the $\gamma$ rays due to natural background, the Ge crystal of each detector was shielded with an annular cylinder of $\mathrm{Pb}$ material having thickness of $3 \mathrm{~cm}$, making the acquitted spectra more fine. The $\gamma$-sources of ${ }^{60} \mathrm{Co},{ }^{133} \mathrm{Ba}$, and ${ }^{152} \mathrm{Eu}$ were used to calculate the absolute efficiency of the detectors. The radioactive sources and the targets were counted in the same geometry.

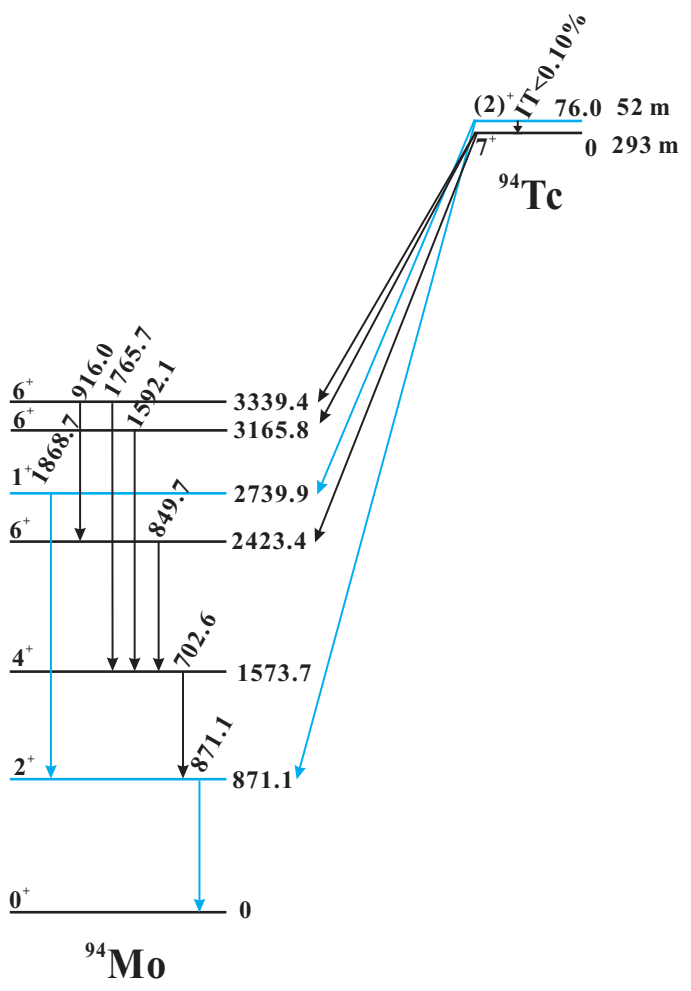

FIG. 1: Decay scheme of ${ }^{94} \mathrm{Tc}$. The data is taken from Ref. [26] , and only partial transitions are shown in the scheme.
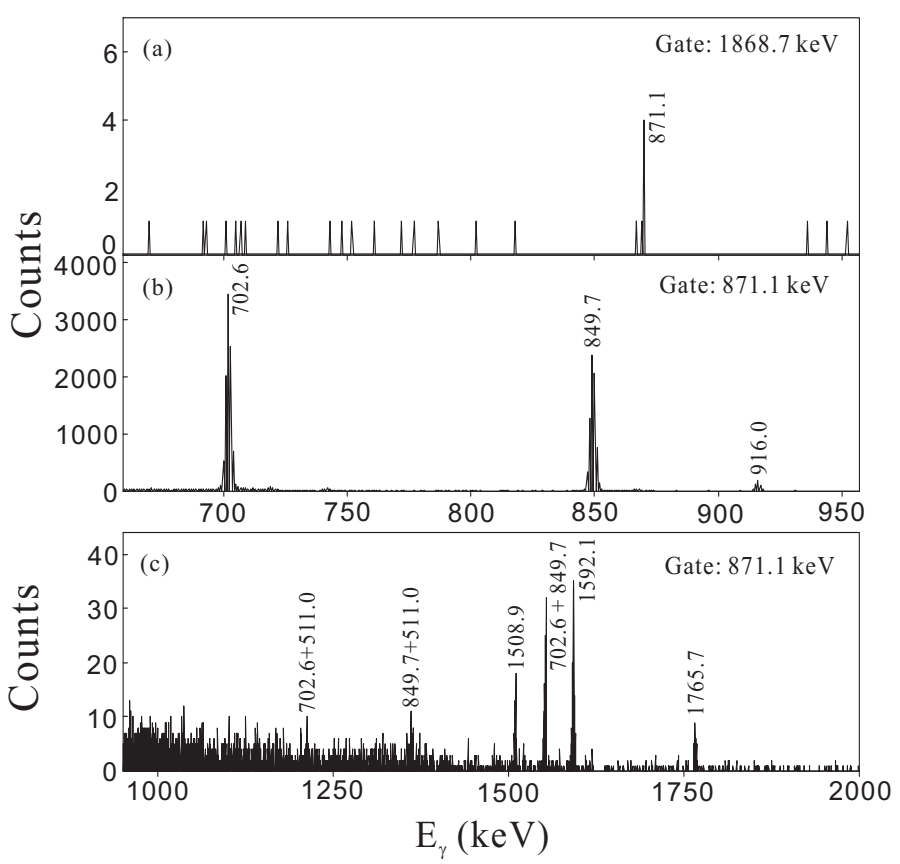

FIG. 2: (Color online) Offline coincidence spectra measured from the decay of ${ }^{94} \mathrm{Tc}$ nucleus, gated by the $1868.7-\mathrm{keV}$ (a) and $871.1-$ $\mathrm{keV}$ (b) \& (c) transitions. 
TABLE I: Measured cross sections of isomeric state $\sigma_{m}$, ground state $\sigma_{g}$, and the isomer ratios $\sigma_{m} / \sigma_{g}$ for the ${ }^{94} \mathrm{Tc}$ formed in the ${ }^{9} \mathrm{Be}+{ }^{89} \mathrm{Y}$ reaction system.

\begin{tabular}{ccccc}
\hline \hline $\mathrm{E}_{l a b}(\mathrm{MeV})$ & $\mathrm{E}_{c . m .} / \mathrm{V}_{B}$ & ${ }^{94} \mathrm{Tc}^{m}(\mathrm{mb})$ & ${ }^{94} \mathrm{Tc}^{g}(\mathrm{mb})$ & $\sigma_{m} / \sigma_{g}\left({ }^{94} \mathrm{Tc}\right)$ \\
\hline 50.1 & 2.14 & $18(3)$ & $463(35)$ & $0.039(8)$ \\
49.1 & 2.09 & $16(3)$ & $460(33)$ & $0.036(6)$ \\
48.1 & 2.05 & $22(2)$ & $419(26)$ & $0.053(7)$ \\
47.0 & 2.01 & $24(2)$ & $412(24)$ & $0.059(5)$ \\
45.9 & 1.96 & $23(3)$ & $427(27)$ & $0.053(7)$ \\
\hline \hline
\end{tabular}

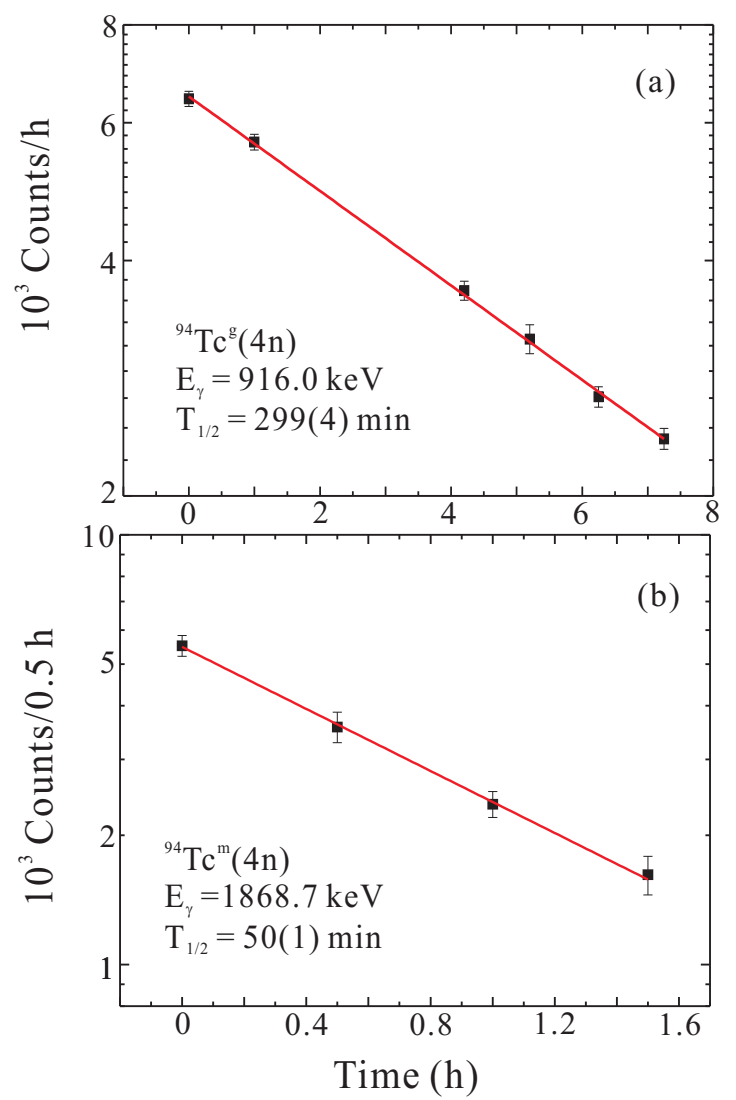

FIG. 3: (Color online) Radioactive decay curves for the (a) ${ }^{94} \mathrm{Tc}^{g}$ and (b) ${ }^{94} \mathrm{Tc}^{m}$ states formed through the ${ }^{9} \mathrm{Be}+{ }^{89} \mathrm{Y}$ reaction by using the 916.0- and $1868.7-\mathrm{keV}$ transitions, respectively. See text for ${ }^{155}$ details.

\section{DATA ANALYSIS AND RESULTS}

The residues of the ${ }^{9} \mathrm{Be}+{ }^{89} \mathrm{Y}$ reaction system covered in the present measurement are mainly from the $3 n, 4 n$, and $5 n$ fusion evaporation channels. Of all these, $4 n$ channel is the dominant one and takes about $70 \%$ of the total cross sections. Fig. 1 shows the decay scheme of the ${ }^{94} \mathrm{Tc}$, taken from

120 Ref. [26]. It can be seen from the decay pattern that the nu- ${ }^{165}$ cleus can decay to ${ }^{94}$ Mo through both $(2)^{+}$isomeric state and $7^{+}$ground state, with different half-lives and characteristic $\gamma$ rays. Therefore, these states can be identified unambiguously. Fig. 2 (a) presents the measured coincidence spectrum gated
125 by the $1868.7-\mathrm{keV}$ transition. The identification of the 871.1$\mathrm{keV}$ transition in the figure clearly proves the decay sequence from the $(2)^{+}$isomeric state. Fig. 2 (b) shows the 871.1-keV gated spectrum, where 702.6-, 849.7-, and 916.0-keV transitions emerge, indicating identification of decay sequence from

130 the $7^{+}$state. The energy range of $871.1-\mathrm{keV}$ gated spectrum was extended to $2000-\mathrm{keV}$ in Fig. 2 (c) to show the weak lines. Other than this, it can also be noted that the 1868.7$\mathrm{keV} \gamma$ ray could not be identified in the $871.1-\mathrm{keV}$ gated spectrum, confirming the dominance of decay sequence from the

${ }_{135} 7^{+}$state. To further justify the identification of the two states in ${ }^{94} \mathrm{Tc}$, the half-lifes of these states i.e., the $7^{+}(916.0-\mathrm{keV}$ line) and $(2)^{+}(1868.7-\mathrm{keV}$ line) are studied via radioactive decay curves as shown in Fig. 3 (a) and 3 (b), respectively. The lifetimes extracted in the present measurement are con40 sistent with the values reported in the literature [26].

The experimental cross sections of ${ }^{94} \mathrm{Tc}$ nuclues in the the ${ }^{9} \mathrm{Be}+{ }^{89} \mathrm{Y}$ reactions were extracted using the half-lives, prominent $\gamma$-ray energies of decay, and intensities following the method described in Refs. [23, 27]. Note that the de-

145 cay of isomeric state by Isomeric Transition (IT) is less than $0.1 \%$ [26], and it affects little on the estimation of the yield of ground state. Fisichella et al. [28] have pointed out the possibility of inaccuracy of a derived excitation function observed mainly in the exponential region of cross section be-

150 low the Coulomb barrier, due to the ambiguities of derived beam energies. In this experiment, the beam energies are well above the barrier, and the changes of reaction cross sections are not dramatic. Therefore, the physics discussion for the present work will not be affected much from the inaccuracy of the adopted beam energies. The extracted cross sections have been tabulated in Table I. The estimation of uncertainties have been made by considering $\approx 3 \%$ error contribution each due to beam intensity, target thickness, and detector efficiency. The systematic uncertainties are added in quadrature to the statistical errors to get the total errors for the present measured cross sections. In practice, we found that the statistical errors make large contribution to the total errors, due to the small branching ratio of decay from the isomeric state.

\section{COMPARISON WITH THEORETICAL CALCULATIONS}

In order to further study the measured isomer yield ratios, we first tried to reproduce the total $\mathrm{CF}$ cross sections. As the ${ }^{9} \mathrm{Be}$ is weakly bound, it may break up before fusing with the 


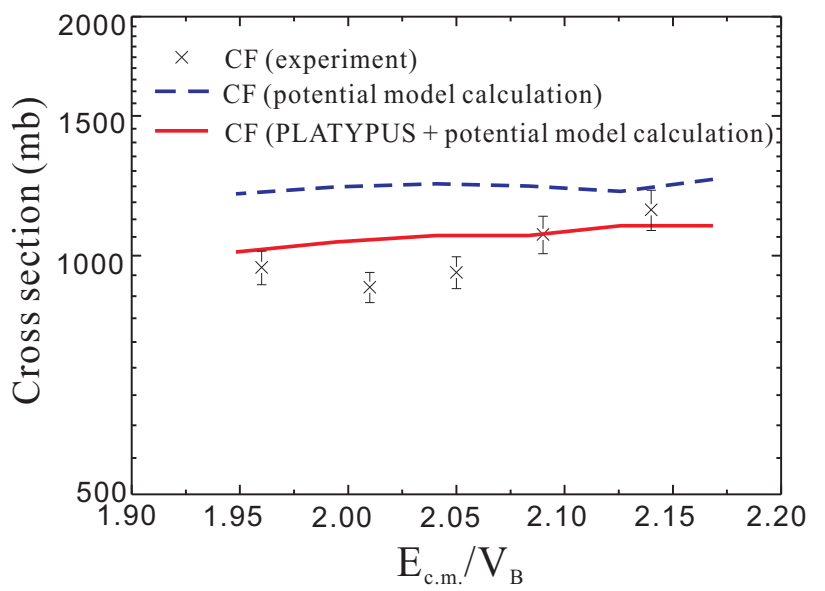

FIG. 4: (Color online) Measured and calculated excitation function ${ }_{195}$ for the complete fusion of the ${ }^{9} \mathrm{Be}+{ }^{89} \mathrm{Y}$ system at above barrier energies. The blue dash line refers to the result from potential model calculation, assuming direct CF of projectile-target without breakup. The red line corresoponds to the result from PLATPUS model combined with potential model calculation, asssuming partial contribution $(34 \%)$ from CF following breakup. The experimental data has ${ }^{200}$ been presented in Ref. [23]. See text for details.

target. In this case, there will be a possibility that the $\mathrm{CF}$ residues resulted from the capture of two ${ }^{4} \mathrm{He}$ particles following the breakup of ${ }^{9} \mathrm{Be}$. This part of residues is calcu- ${ }^{205}$ lated using the PLATYPUS model [22]. Detailed description of the model has been presented in Refs. [29-31]. The other source of the residues considered in the calculation is the direct capture of ${ }^{9} \mathrm{Be}$ without breakup. The associated capture probabilities are obtained from a potential model calculation ${ }^{210}$ by solving the Schrödinger equation with the incoming-waveboundary condition instead of using a sharp cutoff approxi-

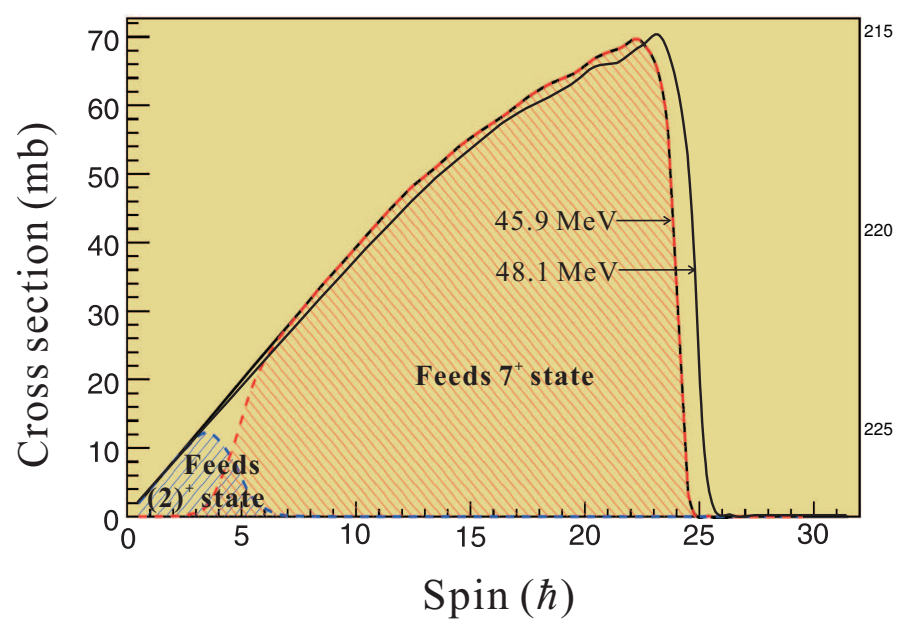

FIG. 5: (Color online) Calculated angular momentum distribution for compound nucleus from $\mathrm{CF}$ of ${ }^{9} \mathrm{Be}+{ }^{89} \mathrm{Y}$ system at beam energies of 45.9- and 48.1-MeV. The division of population between the ground and isomeric state at $45.9-\mathrm{MeV}$ is indicated by the blue and red lines, with $J_{e f f}=4 \hbar$ and $\delta=0.5$. See text for details. mation as employed in the original PLATYPUS model (see e.g., Eq. (4) in Ref. [30] ). In all the calculations, the nuclear interaction potential was determined from the global Broglia-Winther parametrization [32]. The contribution from $\mathrm{CF}$ following breakup to the total $\mathrm{CF}$ was taken as $34 \%$, extracted from the function in Ref. [33]. That function was re185 sulted from the systematically fitting of the measured promptbreakup probabilities. The comparison showing the calculated $\mathrm{CF}$ excitation functions as a function of $\mathrm{E}_{c . m} . / \mathrm{V}_{B}$ has been visualized in Fig. 4 with the experimental data taken from Ref. [23]. Here, $\mathrm{E}_{\text {c.m. }}$. refers to the beam energy, and $\mathrm{V}_{B}$ is the Coulomb barrier energy, both in the center-of-mass frame. One can see from the red line in Fig. 4 that the predicted function is in agreement with the experimental data. We also compared the result assuming no breakup before fusion, determined solely by the potential model calculation, as indicated by the blue line in the figure. Overall, this calculation has overestimated the experimental data, suggesting that the CF following breakup has to be considered in the analysis. Note that we have studied the CF cross sections combining the literature data of above barriers [14, 16], and the necessity of including the ${ }^{9} \mathrm{Be}$ breakups in the full available energy range has been addressed in our previous work [23].

In the fusion evaporation reaction induced by heavy ions, the residues decay more favorably along the yrast lines, therefore the transferred orbital angular momentum in the reaction is supposed to be the main factor affecting the isomer yield ratios. Since in the measured energy region the ${ }^{94} \mathrm{Tc}$ nucleus takes around $70 \%$ of total $\mathrm{CF}$ residues, the $\mathrm{CF}$ compound nucleus was chosen for study, as it could be easily accessed. We have calculated the total spin distribution of the primary compound nucleus, by adding the two sources of CF cross sections mentioned above, namely the capture of two ${ }^{4} \mathrm{He}$ particles following the breakup of ${ }^{9} \mathrm{Be}$ and direct capture of ${ }^{9} \mathrm{Be}$ without breakup. The calculated results for the beam energies of 45.9- and 48.1-MeV are shown in Fig. 5 as examples. To get access to the isomer yield ratios, the phenomenological approach used in Refs. [34, 35] was employed again to analyze the results. The obtained compound nucleus angular momentum distribution was divided into two regions for calculating the relative population of the isomeric state to the ground state with a cutoff angular momentum, $J_{\text {eff }}$. The region with low angular momentum feeds the $(2)^{+}$state, and with high angular momentum feeds the $7^{+}$state. To account for the spread in the angular momentum possibly due to the neutron evaporation, a spreading parameter, $\delta$, was introduced [34]. The isomer yield ratios were calculated as follows:

$$
R=\frac{\sum_{J} \sigma_{J}^{(2)}}{\sum_{J} \sigma_{J}^{(1)}},
$$

$$
\sigma_{J}^{(1)}=\frac{\sigma_{J}}{1+\exp \left(\frac{J_{e f f}-J}{\delta}\right)}
$$




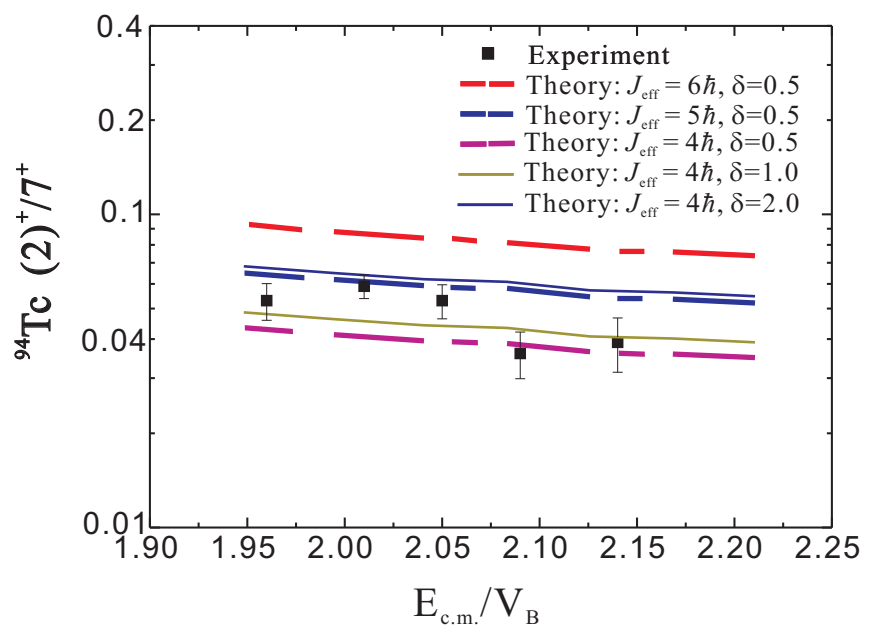

FIG. 6: (Color online) The calculated and measured isomer ratios for ${ }^{94} \mathrm{Tc}$ formed through ${ }^{9} \mathrm{Be}+{ }^{89} \mathrm{Y}$ system, assuming different $J_{\text {eff }}$ and $\delta$ values. See text for details.

$$
\sigma_{J}^{(2)}=\frac{\sigma_{J}}{1+\exp \left(\frac{J-J_{e f f}}{\delta}\right)}
$$

where, $\sigma_{J}$ represents the cross section as a function of the total angular momentum distribution, and $\sigma_{J}{ }^{(1)}$ and $\sigma_{J}^{(2)}$ refer to the cross sections associated with the ground state and isomeric state, respectively [34]. As an example, Fig. 5 also shows the division of population between the ground and isomeric state of CF products at a beam energy of $45.9 \mathrm{MeV}$, with $J_{\text {eff }}=4 \hbar$ and $\delta=0.5$.

As a starting point, to check the dependence of extracted isomer ratio on the cutoff angular momentum $J_{\text {eff }}$, we fixed

the spreading parameter $\delta$ as 0.5 , and calculated the ratios for ${ }^{94} \mathrm{Tc}$ with $J_{\text {eff }}=6,5$, and $4 \hbar$. The results are shown by thick lines in Fig. 6, together with the corresponding experimental data. On comparison, it was observed that the result obtained with $J_{\text {eff }}=4 \hbar$ is more close to the experimental data. To furexplore the dependence of our approach on the spreading parameter, $\delta$ values are varied with 1.0 and 2.0, keeping the $J_{\text {eff }}$ fixed. It revealed that higher $\delta$ gives higher isomer yield ratio. The almost close behaviors of predicted values are observed for the $J_{e f f}=4 \& \delta=2.0$ and $J_{e f f}=5 \& \delta=0.5$. This indicates that in this phenomenological approach neither the fixed cutoff angular momentum nor the fixed spreading parameter is appropriate to describe the data, and only a combination of the two is sufficient. Note that if we fixed the $J_{\text {eff }}$ $=7 \hbar$, which is equal to the spin of ground state, no matter what value is chosen for $\delta$, the predicted isomer yield ratios $_{27}$ are always larger than the experimental data. This suggests that the predicted angular momentum for ${ }^{94} \mathrm{Tc}$ is overall insufficient to reproduce the experimental data. Otherwise, if the predicted angular momentum was higher, there would be more $7^{+}$states supposed to be populated, and in this way the ${ }_{275}$ deduced ratio of $(2)^{+} / 7^{+}$will be lower.

To further test our theoretical approach, the isomer yield

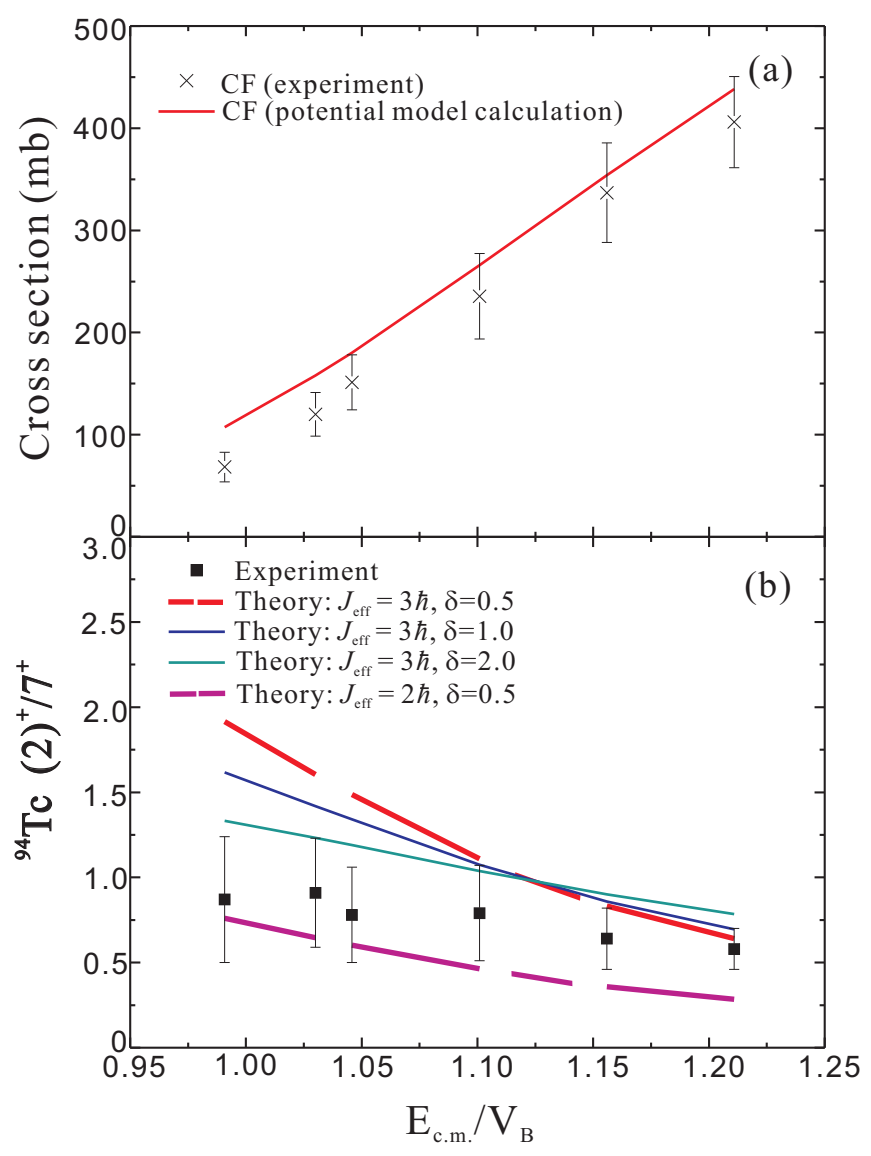

FIG. 7: (Color online) (a) The measured and calculated complete fusion cross sections of the ${ }^{3} \mathrm{He}+{ }^{93} \mathrm{Nb}$ system at above barrier energies. The experimental data is deduced using cross sections from Ref. [36]. The theoretical curve results from potential model calculation. (b) The measured [36] and calculated isomer yield ratios for the ${ }^{94} \mathrm{Tc}$ using different $J_{\text {eff }}$ and $\delta$ values. See text for details.

ratios in ${ }^{94} \mathrm{Tc}$ from ${ }^{93} \mathrm{Nb}\left({ }^{3} \mathrm{He}, 2 \mathrm{n}\right)$ reactions were studied additonally. For this study we chose the energies from Coulomb barrier to $1.2 \mathrm{E}_{c . m} . / \mathrm{V}_{B}$, as in this region the ${ }^{94} \mathrm{Tc}$ is predominant upon all CF residues, thus the easily accessed CF compound nucleus could be selected for study. Fig. 7 (a) presents the comparison of experimental CF cross sections with theoretical predictions. The experimental values were deduced from the total ${ }^{94} \mathrm{Tc}$ cross sections [36], by considering their percentages in CF values from the PACE [37] predictions. The theoretical curve was obtained with potential model calculation, which was adopted to calculate the ${ }^{9} \mathrm{Be}+{ }^{89} \mathrm{Y}$ reaction system as shown in Fig. 4. Note that the breakup of the projectile was not considered in this calculation. The agreement between the theoretical curve and the experimental data in Fig. 7 (a) is good, considering that a global nuclear interaction potential has been used in the fusion calculations. Using a similar approach as that for the ${ }^{9} \mathrm{Be}+{ }^{89} \mathrm{Y}$ system, the deduced isomer yield ratios of ${ }^{94} \mathrm{Tc}$ formed through ${ }^{3} \mathrm{He}+{ }^{93} \mathrm{Nb}$ system are shown in Fig. 7 (b). One can see from the figure that the results obtained from $J_{\text {eff }}=3 \hbar$ and $J_{\text {eff }}=2 \hbar$ are close 
to the experimental data. Adopting a $J_{e f f}=7 \hbar$ will give much higher predictions compared to the experimental value.

This gives the indication again that the predicted angular mo-310 mentum for ${ }^{94} \mathrm{Tc}$ is not enough.

After the compound nucleus was formed in the reaction, the angular momentum of ${ }^{94} \mathrm{Tc}$ would be affected by the emitted neutrons. This maybe one of the reasons that the predictions with $J_{\text {eff }}=7 \hbar$ are higher than the measured isomer yield ${ }_{315}$ ratios. Other than the factors discussed above there also exist some factors which have impacts on the isomer yield ratios in the experiment. For example, the distribution of excitation energy in the compound nucleus may lead to tendentiousness to certain states, the $\gamma$-quantum emitted during the de-320 excitation may shift the angular momentum distribution of the compound nucleus. In these cases, unlike the above adopted phenomenological approach, only the spreading of the angular momentum at a cutoff spin may not be enough. Therefore, the complete knowledge of the decay path of the compound ${ }_{325}$ nuclei is required to accurately describe the isomer ratio data.

\section{SUMMARY}

For the present work, ${ }^{9} \mathrm{Be}+{ }^{89} \mathrm{Y}$ reaction has been used to populate the ${ }^{94} \mathrm{Tc}$ nucleus. The isomer yield ratios have been measured through a standard stacked-foil irradiation by the $e_{330}$ ${ }^{9}$ Be beam followed by offline measurement of the $\gamma$ rays from reaction residues. In the theoretical analysis, the role of $\mathrm{CF}$ following breakup was considered by employing the PLATYPUS model, and the role of direct CF without breakup was demined by potential model calculation. The combination of $f_{335}$ two roles gives an adequate description of experimental excitation functions, even the precise distinction between the two processes could not be established experimentally. In contrast, direct potential model calculation without considering $\mathrm{CF}$ following breakup overestimated the experimental data. By employing a phenomenological approach, the angular momentum distribution has been studied with a cut-off spin and a spreading parameter. The approach managed to reproduce the experimental isomer ratio data qualitatively and in the order of magnitude. We also tested the approach using the isomer yield ratios from ${ }^{3} \mathrm{He}+{ }^{93} \mathrm{Nb}$ system, and obtained similar result. Overall, the predicted angular momentum is insufficient to accurately reproduce the experimental data. More precise knowledge is still needed to completely describe the decay path of the compound nucleus, the spread of angular momentum, and ultimately the measured isomer yield ratios. The analysis of the ${ }^{9} \mathrm{Be}+{ }^{89} \mathrm{Y}$ and ${ }^{3} \mathrm{He}+{ }^{93} \mathrm{Nb}$ systems offers the opportunity to further compare other projectile-target combinations, and deeply understand the reaction mechanisms. This work also extends information on the production of ${ }^{94} \mathrm{Tc}$ nucleus - one of the promising candidates in the application of medical diagnostics.

\section{ACKNOWLEDGEMENTS}

The work at Institute of Modern Physics, CAS was supported by the Youth Innovation Promotion Association of Chinese Academy of Sciences (Grant No. 2019407), the Strategic Priority Research Program of Chinese Academy of Sciences (Grant No. XDB34010000), and the National Natural Science Foundation of China (Grants No. U1932138, No. U1832134, No. 11775274, and No. 11305221). The work at University of Surrey was supported by the STFC (Grant No. ST/P005314/1).
[1] T. J. Ruth, Reports on Progress in Physics 72, 016701 (2008), URL https://doi.org/10.1088\%2F0034-4885\% $2 \mathrm{~F} 72 \div 2 \mathrm{~F} 1 \div 2 \mathrm{~F} 016701$

[2] T. Hayakawa, Y. Hatsukawa, and T. Tanimori, Heliyon 4, e00497 (2018), ISSN 2405-8440, URL365 http://www.sciencedirect.com/science/ article/pii/s2405844017330700.

[3] C. Hoehr, T. Morley, K. Buckley, M. Trinczek, V. Hanemaayer, P. Schaffer, T. Ruth, and F. Bénard, Applied Radiation and Isotopes 70, 2308 (2012), ISSN 0969-8043,370 URL http://www.sciencedirect.com/science/ article/pii/s0969804312003697.

[4] K. Gagnon, S. McQuarrie, D. Abrams, A. J. McEwan, and F. Wuest, Current Radiopharmaceuticals 4, 90 (2011), ISSN 1874-4710/1874-4729, URL http://WWW.375 eurekaselect.com/node/94481/article.

[5] B. T. Christian, R. J. Nickles, C. K. Stone, T. L. Mulnix, and J. Clark, Applied Radiation and Isotopes 46, 69 (1995), ISSN 0969-8043, URL http://www.sciencedirect. com/science/article/pii/096980439400130R. 380

[6] J. J. Hogan, Journal of Inorganic and Nuclear Chemistry 35, 705 (1973), ISSN 0022-1902, URL http: //wWw.sciencedirect.com/science/article/ pii/0022190273804373.

[7] G. Galy, B. Philippon, A. Bardy, and R. Munsch, The International Journal of Applied Radiation and Isotopes 32, 277 (1981), ISSN 0020-708X, URL http://www.sciencedirect.com/science/ article/pii/0020708X81900855.

[8] M. Izumo, H. Matsuoka, T. Sorita, Y. Nagame, T. Sekine, K. Hata, and S. Baba, International Journal of Radiation Applications and Instrumentation. Part A. Applied Radiation and Isotopes 42, 297 (1991), ISSN 0883-2889, URL http://www.sciencedirect.com/ science/article/pii/088328899190092F.

[9] F. Tárkányi, F. Ditrói, A. Hermanne, S. Takács, and A. Ignatyuk, Nuclear Instruments and Methods in Physics Research Section B: Beam Interactions with Materials and Atoms 280, 45 (2012), ISSN 0168-583X, URL http://www.sciencedirect.com/science/ article/pii/s0168583X12001437.

[10] B. Strohmaier, M. Faßbender, and S. M. Qaim, Phys. Rev. C 56, 2654 (1997), URL https: / / link.aps.org/doi/ $10.1103 /$ PhysRevC.56.2654.

[11] J. Zaidi, M. Arif, S. Ahmed, and I. Qureshi, Radiochimica Acta 85, 9 (1999), URL https://www.scopus.com/ 
inward/record.uri?eid=2-s2.0-0002332798\& 435 do $i=10.1524 \% 2$ fract .1999 .85 .12 .9 \&partner $I D=$ $40 \& \mathrm{md} 5=08 \mathrm{~d} 7 \mathrm{f} 57583 \mathrm{f} 1144 \mathrm{a} 5853 \mathrm{~d} 9 \mathrm{ffac} 307008$.

[12] J. Zaidi, M. Arif, I. Fatima, S. Waheed, S. Ahmad, and I. Qureshi, Radiochimica Acta 93, 547 (2005), cited By 6, URL https://www.scopus.com/inward/record.440 uri?eid=2-s2.0-27744487000\&doi=10.1524\% 2 fract $.2005 .93 .9-10.547 \&$ partner ID $=40$ \&md5= $02 \mathrm{c} 2 \mathrm{~d} 608525850616 \mathrm{edd} 1 \mathrm{c} 3 \mathrm{f} 17932 \mathrm{c} 4 \mathrm{~d}$.

[13] K. Gagnon, F. Bénard, M. Kovacs, T. J. Ruth, P. Schaffer, J. S. Wilson, and S. A. McQuarrie, Nuclear Medicine 445 and Biology 38, 907 (2011), ISSN 0969-8051, URL http://www.sciencedirect.com/science/ article/pii/s0969805111000473.

[14] M. Maiti and S. Lahiri, Phys. Rev. C 81, 024603 (2010), URL https:// link . aps . org/doi/10.1103/450 PhysRevC.81.024603.

[15] B. B. Kumar, S. Mukherjee, S. Chakrabarty, B. S. Tomar, A. Goswami, and S. B. Manohar, Phys. Rev. C 57, 743 (1998), URL https://link.aps.org/doi/10.1103/ PhysRevC.57.743.

[16] C. S. Palshetkar, S. Santra, A. Chatterjee, K. Ramachandran, S. Thakur, S. K. Pandit, K. Mahata, A. Shrivastava, V. V. Parkar, and V. Nanal, Phys. Rev. C 82, 044608 (2010), URL https: //link .aps.org/doi/10.1103/ PhysRevC.82.044608.

[17] A. Diaz-Torres and I. J. Thompson, Phys. Rev. C 65, 024606 (2002), URL https://link.aps.org/doi/10.1103/ PhysRevC.65.024606.

[18] A. Diaz-Torres, I. J. Thompson, and C. Beck, Phys. Rev. C 68, 044607 (2003), URL https: / / ink . aps.org/doi/465 10.1103/PhysRevC.68.044607.

[19] I. J. Thompson and A. Diaz-Torres, Progress of Theoretical Physics Supplement 154, 69 (2004), ISSN 0375-9687, https://academic.oup.com/ptps/articlepdf/doi/10.1143/PTPS.154.69/5227135/154-69.pdf, URL470 https://doi.org/10.1143/PTPS.154.69.

[20] K. Yabana, Progress of Theoretical Physics 97, 437 (1997), ISSN 0033-068X, https://academic.oup.com/ptp/articlepdf/97/3/437/5205631/97-3-437.pdf, URL https: //doi.org/10.1143/PTP.97.437.

[21] M. Boselli and A. Diaz-Torres, Phys. Rev. C 92, 044610 (2015), URL https://link.aps.org/doi/10.1103/ PhysRevC.92.044610.

[22] A. Diaz-Torres, Computer Physics Communications 182, 1100 (2011).

[23] G. S. Li, M. L. Liu, D. Patel, Y. D. Fang, X. H. Zhou, Y. H. Zhang, A. Diaz-Torres, C. S. Palshetkar, J. Lubian, N. T. Zhang, et al., Phys. Rev. C 101, 014606 (2020), URL https://link .aps.org/doi/10.1103/ PhysRevC.101.014606.
[24] D. Bazin, O. Tarasov, M. Lewitowicz, and O. Sorlin, Nuclear Instruments and Methods in Physics Research Section A: Accelerators, Spectrometers, Detectors and Associated Equipment 482, 307 (2002), ISSN 0168-9002, URL http://www.sciencedirect.com/ science/article/pii/s0168900201015042.

[25] C. Scheidenberger and H. Geissel, Nuclear Instruments and Methods in Physics Research Section B: Beam Interactions with Materials and Atoms 135, 25 (1998), ISSN 0168-583X, URL http://www.sciencedirect.com/ science/article/pii/s0168583X97006393.

[26] D. Abriola and A. Sonzogni, Nuclear Data Sheets 107, 2423 (2006), ISSN 0090-3752, URL http: //www.sciencedirect.com/science/article/ pii/s0090375206000652.

[27] N. T. Zhang, Y. D. Fang, P. R. S. Gomes, J. Lubian, M. L. Liu, X. H. Zhou, G. S. Li, J. G. Wang, S. Guo, Y. H. Qiang, et al., Phys. Rev. C 90, 024621 (2014), URL https://link. aps.org/doi/10.1103/PhysRevC.90.024621.

[28] M. Fisichella, A. C. Shotter, A. Di Pietro, P. Figuera, M. Lattuada, C. Marchetta, V. Privitera, L. Romano, C. Ruiz, and M. Zadro, Phys. Rev. C 92, 064611 (2015), URL https://link.aps.org/doi/10.1103/ PhysRevC.92.064611.

[29] A. Diaz-Torres, D. Hinde, J. A. Tostevin, M. Dasgupta, and L. Gasques, Physical Review Letters 98, 152701 (2007).

[30] A. Diaz-Torres, Journal of Physics G: Nuclear and Particle Physics 37, 075109 (2010).

[31] A. Diaz-Torres and D. Quraishi, Physical Review C 97, 024611 (2018).

[32] W. Reisdorf, Journal of Physics G: Nuclear and Particle Physics 20, 1297 (1994), URL https://doi.org/10. $1088 \div 2 \mathrm{~F} 0954-3899 \div 2 \mathrm{~F} 20 \div 2 \mathrm{~F} 9 \div 2 \mathrm{~F} 004$.

[33] B. Wang, W.-J. Zhao, A. Diaz-Torres, E.-G. Zhao, and S.-G. Zhou, Phys. Rev. C 93, 014615 (2016), URL https://link.aps.org/doi/10.1103/PhysRevC. 93.014615 .

[34] L. Gasques, M. Dasgupta, D. Hinde, T. Peatey, A. Diaz-Torres, and J. Newton, Physical Review C 74, 064615 (2006).

[35] G. S. Li, Y. D. Fang, A. Diaz-Torres, M. L. Liu, N. T. Zhang, X. H. Zhou, Y. H. Zhang, J. G. Wang, B. S. Gao, Y. H. Qiang, et al., Phys. Rev. C 99, 054617 (2019), URL https://link .aps.org/doi/10.1103/ PhysRevC.99.054617.

[36] M. Fa $\beta$ bender, A. F. Novgorodov, F. Rösch, and S. M. Qaim, Radiochimica Acta 65, 215 (01 Jun. 1994), URL https://www. degruyter.com/view/journals/ ract /65/4/article-p215. xml.

[37] A. Gavron, Phys. Rev. C 21, 230 (1980), URL https: // link.aps.org/doi/10.1103/PhysRevC.21.230. 